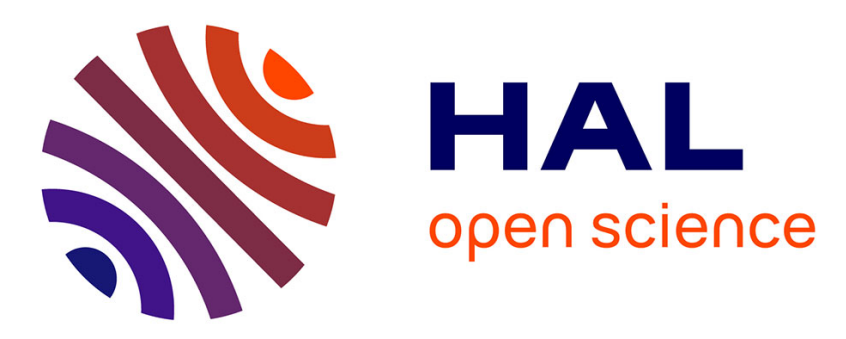

\title{
A novel method for the measurement of elastic moduli of fibres
}

Didier Perrin, Martin Alteirac, Stéphane Corn, Martin E.R. Shanahan

\section{To cite this version:}

Didier Perrin, Martin Alteirac, Stéphane Corn, Martin E.R. Shanahan. A novel method for the measurement of elastic moduli of fibres. Composites Part A: Applied Science and Manufacturing, 2007, 38 (1), pp.71-79. 10.1016/j.compositesa.2006.01.013 . hal-02536535

\section{HAL Id: hal-02536535 \\ https://hal.mines-ales.fr/hal-02536535}

Submitted on 17 Apr 2020

HAL is a multi-disciplinary open access archive for the deposit and dissemination of scientific research documents, whether they are published or not. The documents may come from teaching and research institutions in France or abroad, or from public or private research centers.
L'archive ouverte pluridisciplinaire HAL, est destinée au dépôt et à la diffusion de documents scientifiques de niveau recherche, publiés ou non, émanant des établissements d'enseignement et de recherche français ou étrangers, des laboratoires publics ou privés. 


\title{
A novel method for the measurement of elastic moduli of fibres
}

\author{
Didier Perrin, Martin Alteirac, Stéphane Corn, Martin E.R. Shanahan * \\ Centre des Matériaux (CMGD), Ecole des Mines d'Alès, 6 Avenue de Clavière, 30319 Alès Cedex, France
}

\begin{abstract}
The elastic modulus of fibres used in composite materials is a parameter of prime importance in the determination of overall mechanical behaviour. However, evaluation of Young's modulus, $E$, of a fibre is a delicate operation given the small dimensions (diameter typically a few tens of microns), and therefore low forces involved in tensile testing. This article treats a novel method of modulus assessment involving the bending of fibres, clamped at one extremity, by forced vibrations. The fibre behaves as a beam, and when the forced oscillations approach (one of) the resonant frequency(ies) of the fibre, the bending amplitude increases. Classical beam theory allows evaluation of Young's modulus from knowledge of resonant frequency, and fibre dimensions and density. Preliminary application of the technique using fibres of $E$-glass, having well known elastic characteristics, has given good results and shown its inherent potential. Subsequently, the technique developed was used on recycled fibres in order to obtain their Young's modulus and to assess their loss of mechanical properties when compared to virgin fibres.
\end{abstract}

Keywords: A. Fibres; A. Recycling; B. Fracture; B. Mechanical properties

\section{Introduction}

Many structural composite materials benefit from weight saving due to the use of a low-density, resilient polymeric matrix, combined with relatively high elastic modulus, endowed by the inclusion of fibre fillers, generally presenting quite a high intrinsic Young's modulus, $E$. The mechanisms by which added stiffness is achieved are now legend, be this for long, or short, fibre composites [1-3]. A major (although not the unique) parameter conferring stiffness is the elastic modulus of the fibrous component. However, many frequently-used fibres are of small diameter, of the order of tens of microns, thus precluding simple stressstrain measurements because of the necessary delicate manipulation and small tensile forces required to test such small cross-sectional bodies without premature fracture.

\footnotetext{
* Corresponding author. Present address: Laboratoire de Mécanique Physique (LMP) - UMR CNRS 5469, Université Bordeaux 1, 351 Cours de la Libération, 33405 TALENCE Cedex, France. Tel.: +3346678 53 54; fax: +33466785365 .

E-mail address: Martin.Shanahan@ema.fr (M.E.R. Shanahan).
}

To present, one of the most successful techniques indeed involves direct tensile tests, using precision-built equipment [4-7]. However, due to the difficulty of successfully attaching both fibre ends, as is necessary in tension, and allowing for the consequent, inevitable perturbing effects on measurement, a variety of gauge lengths is often used in order to improve precision, by extrapolating to infinite length.

Thus, when endeavouring to obtain the elastic modulus of fibres, it is often the measurement of true strain, rather than applied load, which poses greater problems. Clearly, non-contact strain measurement techniques are superior in this respect. Such methods exist, but are in general expensive. Non-contact strain measurement methods include, for instance, Raman spectroscopy and laser extensometry.

Micro-Raman spectroscopy, with its high spatial resolution $(0.1-1 \mu \mathrm{m})$, is possibly one of the most suitable methods for measuring strain in individual fibres and determining the transverse modulus, since the average diameter of the fibres is generally greater than the spatial resolution of the micro-Raman spectroscopy [8]. Additionally, Raman Spectroscopy has excellent wave number precision and it is also possible to conduct non-destructive, 
non-contact and short-time measurement near the surface in a micro-area of Raman-active material. An example is given in [9]. A thin film of $\mathrm{PbO}$ was deposited, by resistance heating PVD, on the outer surface of the fibre specimen and a laser beam focused to give a spot size of approximately $1 \mu \mathrm{m}$ diameter on the fibre to measure transverse strain in order to determine its modulus.

A different approach, referred to as the Raman coating method, relies on the measurement of strain in Ramanactive thin films applied to fibres, thus enabling the strain of Raman-inactive composites to be observed by Raman spectroscopy [10].

As for laser extensometry, one technique developed involves the mounting of an extensometer equipped with an infrared lamp on a standard servo-hydraulic fatigue testing machine [11]. The principle of the technique consists of applying a sinusoidal time-dependent stress, $\sigma=\sigma_{0}$. $\sin \omega t$, to the specimen, leading to a resulting strain, $\varepsilon=\varepsilon_{0} \sin (\omega t-\varphi)$, where $\varepsilon_{0}$ and $\varphi$, respectively, amplitude and phase lag, are measured. The amplitude and phase data were obtained and treated using a frequency response analyser.

The technique presented here, which is new to the authors' knowledge, largely eliminates the inherent problems related to tensile testing on this minute scale. Instead of straining an individual fibre in tension, the fibre is bent, thus only one extremity is in need of attachment. In addition, the solitary physical connection to the external apparatus only experiences lateral forces, to all intents and purposes, and so the fibre/ support interface is under a low level of stress.

\section{Theory}

The basis of the method developed here is to consider a fibre clamped at one extremity as behaving as a cantilever beam, encastré at one end, of length, $l$, and sectional moment of inertia, $I$. Since we shall be treating fibres of essentially circular section of radius, $r$, we have, with respect to the longitudinal plane of symmetry, $I=\pi r^{4} / 4$ (see Fig. 1, but note that the aspect ratio, $l / r$, is typically much greater than that portrayed in the figure, for clarity). The basic theory of oscillations of such a beam is classical and may be found in standard treatises on vibrational mechanics, e.g. [12-15]. Suffice it in this context to give a succinct outline derivation.

In Fig. 1, $x$ represents distance along the (undeformed) fibre from the origin, $O$, at the point of fixation, and $y$ is the fibre displacement normal to $x$. The problem is assumed to be 2-dimensional. Since the system is oscillatory, and for our purposes in a steady state regime, we assume that separation of variables is permitted:

$y(x, t)=z(x) \cdot \sin (2 \pi v t+\zeta)$,

$\frac{\partial y}{\partial t}=\dot{y}=2 \pi v \cdot z(x) \cdot \cos (2 \pi v t+\zeta)$,

...etc.,

where $v$ is frequency, $t$ is time and $\zeta$ is simply an arbitrary phase angle.

The Lagrangian function for the oscillating beam, $L$, is composed of the difference between kinetic, $T$, and potential, $V$, (i.e. elastic, stored) energies, and can be written as:

$$
\begin{aligned}
L\left(y, y^{\prime}, y^{\prime \prime}, \dot{y}, \dot{y}^{\prime}\right) & =T\left(y, y^{\prime}, \dot{y}, \dot{y}^{\prime}\right)-V\left(y, y^{\prime}, y^{\prime \prime}\right) \\
& =\int_{0}^{l}\left(\frac{1}{2} m \dot{y}^{2}-\frac{1}{2} E I y^{\prime 2}\right) \mathrm{d} x,
\end{aligned}
$$

where $m$ is the linear density of the fibre (mass/unit length) and $E$ its Young's modulus. The dot and prime have their usual meanings of differentiation with respect to $t$ and $x$.

Following classical Lagrangian mechanics principles and applying the Euler variational equation to expression (3), after considerable algebra, it can be found that $y(x, t)$ must obey:

$y^{\prime \prime \prime \prime}+\frac{m}{E I} \ddot{y}=0$.

Inspired by Eqs. (1) and (2) et seq., separation of variables leads to:

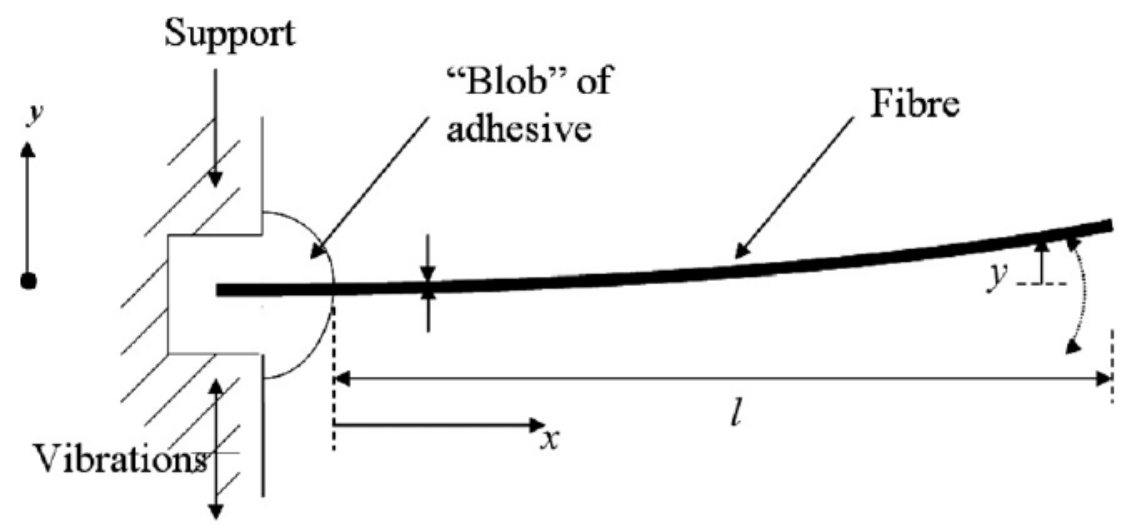

Fig. 1. Schematic representation of fibre embedded in "blob" of adhesive attached to support, and nomenclature employed (NB: aspect ratio, $l / r$, is in reality typically much greater than shown: see Fig. 2). 
$\frac{\mathrm{d}^{4} z}{\mathrm{~d} x^{4}}-\gamma^{4} z=0$,

where

$\gamma=\left(4 \pi^{2} m v^{2} / E I\right)^{1 / 4}$.

Eq. (5) is solved using the boundary conditions of

$z(0)=\frac{\mathrm{d} z}{\mathrm{~d} x}(0)=0$

and

$\frac{\mathrm{d}^{2} z}{\mathrm{~d} x^{2}}(l)=\frac{\mathrm{d}^{3} z}{\mathrm{~d} x^{3}}(l)=0$.

Again, after a significant amount of algebra, we obtain the identity:

$\cos (\gamma l) \cdot \cosh (\gamma l)+1=0$

which has the (iterative, or graphical) solutions of $\gamma l=1.875$ for the first harmonic solution $(n=1)$, and $\gamma l=4.694$ for the second mode $(n=2)$. Thus, from Eq. (6), considering specifically the former solution, for $n=1$, the characteristic (primary) frequency of vibration is given by:

$v_{1}=\frac{1.875^{2}}{2 \pi l^{2}}\left(\frac{E I}{m}\right)^{1 / 2}$

and this value of $v_{1}$ corresponds to resonance. Alternatively, assuming a fibre of circular section $\left(I=\pi r^{4} / 4\right)$, and rearranging, we obtain:

$E=12.8 \frac{l^{4} \rho}{r^{2}} \cdot v_{1}^{2}$,

for the fundamental mode. Equivalently:

$E=0.325 \frac{l^{4} \rho}{r^{2}} \cdot v_{2}^{2}$,

for the second mode, with resonant frequency, $v_{2}$. In Eqs. (11) and (12), $\rho$ is the density of the material constituting the fibre, as opposed to the linear density $\left(\rho=m / \pi r^{2}\right)$.

Eq. (11) thus gives Young's modulus of the vibrating fibre from a knowledge of its fundamental resonant frequency, dimensions and density, and Eq. (12) from its second harmonic. This means that if the fibre, retained in the beam configuration described, is made to vibrate by external means applied to its support, perpendicularly to the fibre length, and this external frequency is varied by scanning, a marked increase in displacement amplitude should be expected for frequencies, $v_{n}$, satisfying Eqs. (11) and (12) (and in principle for higher modes, $n \geqslant 3$, where the prefactor will be smaller, but these will be harder to attain experimentally). The above treatment assumes no energy dissipation effects on fibre motion; a point which will be discussed later.

\section{Materials and techniques}

\subsection{Materials}

The model fibres used in the development of the present work were made of the well-known $E$-glass [16], manufactured by Vetrotex St Gobain International. Commercial short glass fibres have an average length of $4.5 \mathrm{~mm}$. This material has a well-established Young's modulus of $73 \pm$ $2 \mathrm{GPa}$, in its normal state, and a density, $\rho$, of $2600 \pm$ $100 \mathrm{~kg} \mathrm{~m}^{-3}$. Fibre diameters are typically approximately $16 \mu \mathrm{m}$ [17]. The virgin fibres used here, having been cut specifically, had lengths, $l$, in the range of approximately $1.8-13 \mathrm{~mm}$. The dimensions of individual fibres were obtained using both optical and, more successfully, scanning electron microscopy (Quanta 200 FEG, FEI Company). Homogeneity of fibre cross-sections was also verified, by measurement at different places. With rare exceptions, the standard deviation of fibre radii was found to be approximately $0.5 \mu \mathrm{m}$ or less.

After having first developed the present technique on virgin fibres, used fibres, obtained from sheet-moulding compound (SMC), were also studied. Panels of SMC composite of dimensions $500 \times 500 \times 2 \mathrm{~mm}^{3}$ were cut up and then shredded with a Rotoplex-Alpine Rotary Cutter Mill turning at $1500 \mathrm{rpm}$ for $25 \mathrm{~min}$, to obtain model SMC production waste. This material contains $E$-glass fibres representing a quarter of the composition of the SMC. The passing grounds were recuperated after sieving with a $315 \mu \mathrm{m}$ wire gauze. Three successive treatments of fibres were undertaken and the resulting Young's modulus following each stage assessed by the vibration technique described above.

- shredding and sieving only (1st treatment) [18],

- shredding and dissolving the passing ground in a buffer acid solution of $\mathrm{H}_{3} \mathrm{PO}_{4}$ at $\mathrm{pH} 2.0$ (2nd treatment) [18],

- shredding, dissolution and finally thermal treatment of the SMC grounds at $200{ }^{\circ} \mathrm{C}$ for $3 \mathrm{~h}$ (3rd treatment).

After all three stages of treatment, clean, straight fibres were chosen for testing.

In initial tests, the fibres to be characterised were individually attached to flat polymeric (cellulose acetate) supports, perpendicularly to their axes, by means of a small "blob" of Araldite ${ }^{\circledast}$ adhesive. For later tests, the polymeric supports were replaced by steel, but similar geometry was retained (Section 4). The adhesive was positioned in and around a small hole previously drilled in the support $(\approx 3$ $\mathrm{mm}$ in diameter and $2 \mathrm{~mm}$ in depth) to enhance the rigidity of the mounting. Fig. 2 gives a typical microscopic view of a fibre thus prepared, embedded in its adhesive "blob". Fibre length, $l$, was measured from the top of the adhesive "blob" to the free fibre end: the (relatively) massive dimensions of the "blob" compared to the fibre, and the fact that the adhesive surface was virtually perpendicular to the fibre axis imply that any contribution to vibration of the fibre 


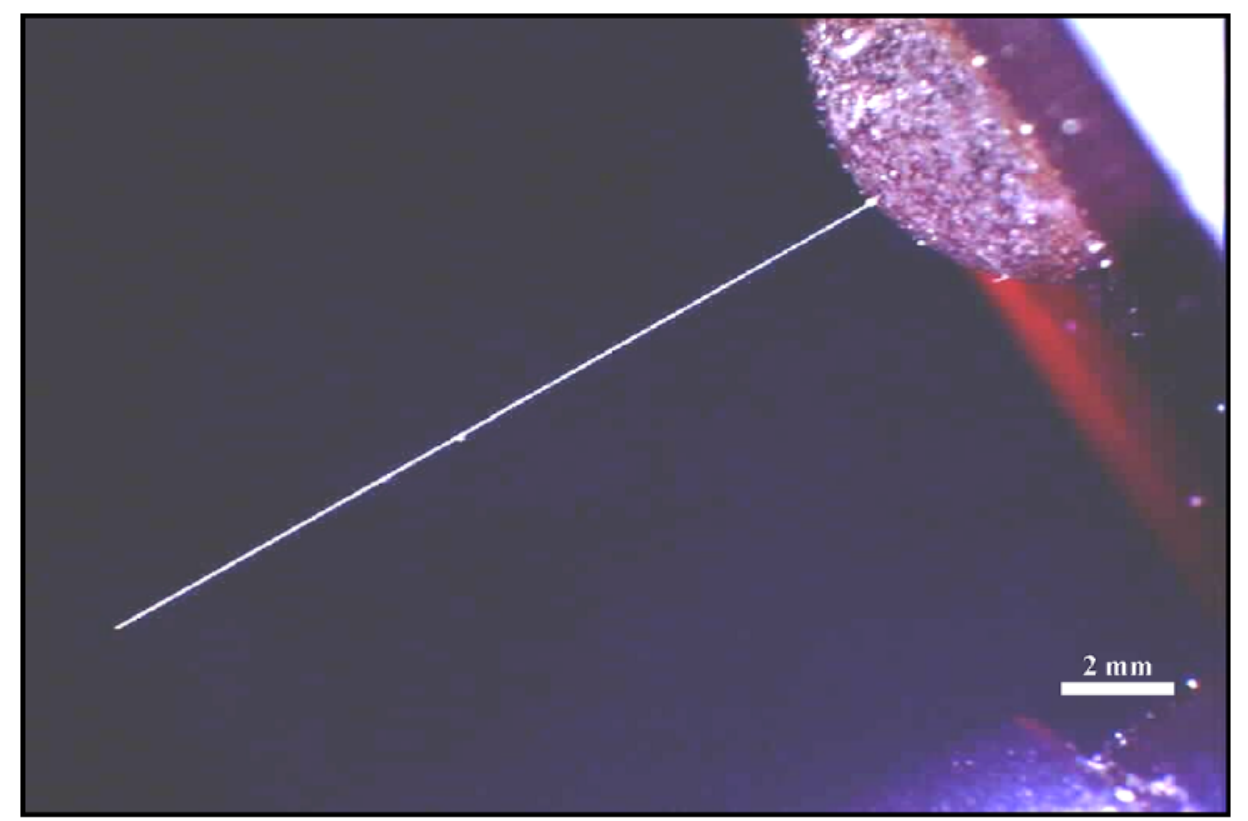

Fig. 2. E-glass fibre of length approximately $13 \mathrm{~mm}$ embedded in adhesive "blob" on cellulose acetate support.

beneath the adhesive surface will be totally negligible. (It was fortunate that the adhesive did not wet the fibre too much (high contact angle), and thus "climb" up the fibre shaft: this would have rendered the initial part of the fibre, near the adhesive, a "composite material", invalidating the hypothesis of constant cross-section and modulus.) It is perhaps worth noting that the simplification adopted would probably not be valid were the fibre to be tested in tension, parallel to its axis, as opposed to perpendicularly, as is the case here.

\subsection{Experimental techniques}

Individual fibres, of various lengths, each mounted on its support, were attached to the vibrating platform of an electrodynamic shaker (Ling Dynamic System), in series with a sinusoidal wave power supply and amplifier (Hameg Instrument), capable of imposing oscillations in the range $0.3-9000 \mathrm{~Hz}$. Fibre assemblies were attached using a thin layer of organic wax on the area of the vibration platform.

Attachment was assured so that the vertical oscillations of the electrodynamic shaker platform, and therefore fibre support, were perpendicular to the horizontal fibre axis leading to flexural beam-type movement of the fibre, due to inertial effects (see Fig. 1). Starting generally from low frequencies, the range was scanned slowly, with measurements being taken near fibre resonance windows. Experiments were undertaken in still air at approximately $25^{\circ} \mathrm{C}$ and ambient humidity ( $\approx 50 \% \mathrm{RH})$.

Different configurations were tried in order to observe the vibrating fibre and its support, based mainly on having a source of illumination behind the fibre, a bi-convex lens in front, followed by a screen, typically with distances of approximately $0.5 \mathrm{~m}$ between fibre and lens and $2.5 \mathrm{~m}$ between lens and screen. With the set-up employed, magnification was of the order $100 \times$. To facilitate observation, experiments took place in a darkroom. The overall experimental set-up for fibre vibration and monitoring is shown schematically in Fig. 3.

\section{Results}

Preliminary tests on virgin fibres effected with polymeric supports led to satisfactory estimates of Young's modulus, in good agreement with the accepted value and in line with those discussed below obtained using steel supports, yet rather more scatter was observed. This was at least in part due to the wider resonance peaks (Section 5). For this reason, we opted for the steel supports for the major part of this study, and the quantitative treatment and discussion below concern these. Table 1 summarises data obtained (with steel supports), and necessary to compare predicted and experimentally obtained values of $E$, the latter being calculated from Eqs. (11) and (12) and respective values of resonant frequency, $v$. Preliminary calculations, based on the accepted values for $E$ and $\rho$ for $E$-glass given above, showed that the fundamental mode (in brackets: mode 2) resonance should occur at a frequency of approximately $3 \mathrm{kHz}(26 \mathrm{kHz})$ for fibres of length of approximately $1.8 \mathrm{~mm}$, decreasing to approximately $70 \mathrm{~Hz}(460 \mathrm{~Hz})$ for $l \approx 13 \mathrm{~mm}$. It can be seen that the tabulated values corresponding to these two extremes of the range of fibre lengths studied are in good agreement with these estimates.

In Fig. 4, we show a graphical interpretation of the results given in Table 1. Young's modulus, $E$, is given as the ordinate, with fibre length, $l$, as the abscissa. Values of $E$ have been estimated from both vibration modes, using Eqs. (11) and (12). Also in this figure, we have added the 


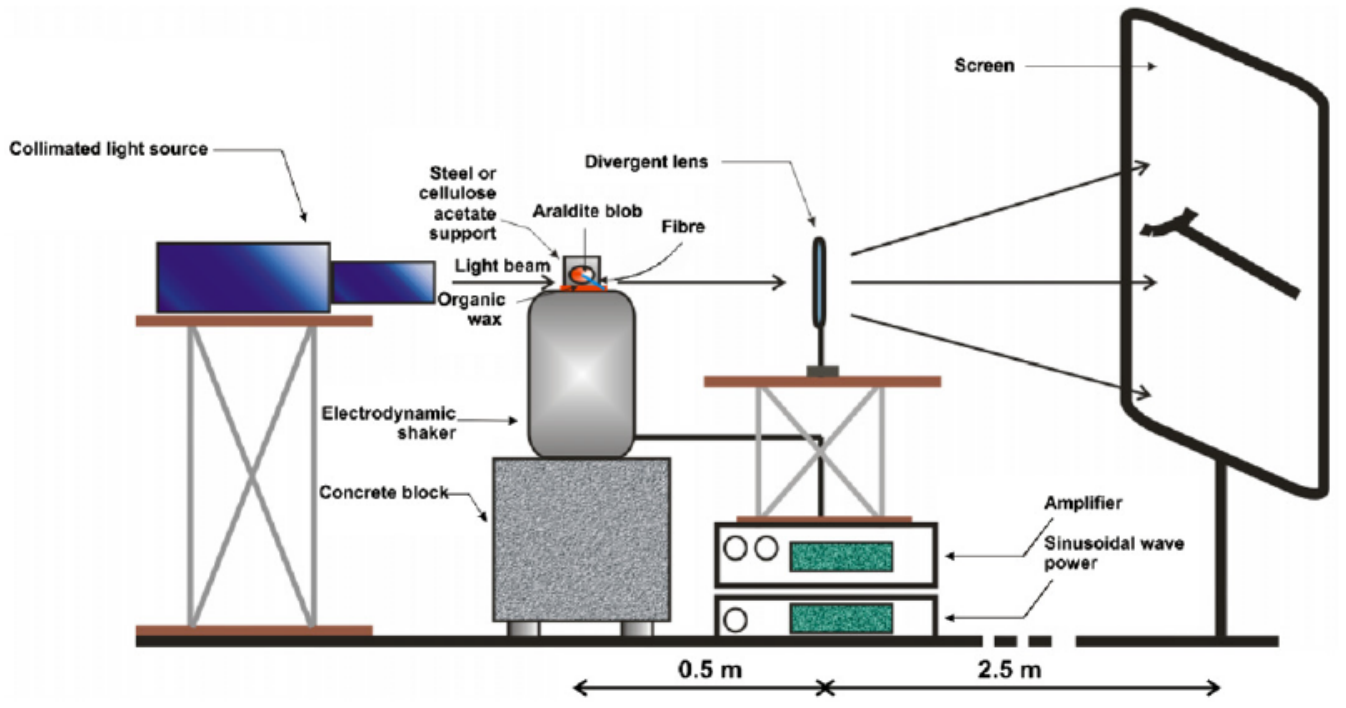

Fig. 3. Schematic representation of overall experimental set-up for fibre vibration and monitoring.

Table 1

Lengths, $l$, of virgin $E$-glass fibres tested, and their corresponding measured resonant frequencies for both fundamental and secondary modes, $v_{1}$ and $v_{2}$, and estimated errors

\begin{tabular}{|c|c|c|c|c|c|c|}
\hline \multirow[t]{2}{*}{$\overline{l(\mathrm{~mm})}$} & \multicolumn{3}{|c|}{ Fundamental mode } & \multicolumn{3}{|c|}{ Second mode } \\
\hline & $v_{1}(\mathrm{~Hz})$ & $E_{1}(\mathrm{GPa})$ & $\frac{E_{1}-E}{E} \times 100(\%)$ & $v_{2}(\mathrm{~Hz})$ & $E_{2}(\mathrm{GPa})$ & $\frac{E_{2}-E}{E} \times 100(\%)$ \\
\hline $1.77 \pm 0.01$ & $3760 \pm 5$ & 72.9 & -0.14 & - & - & - \\
\hline $2.39 \pm 0.02$ & $2090 \pm 5$ & 74.7 & 2.33 & - & - & - \\
\hline $4.91 \pm 0.01$ & $490 \pm 5$ & 73.4 & 0.55 & $2855 \pm 5$ & 63.2 & -13.42 \\
\hline $6.71 \pm 0.01$ & $260 \pm 5$ & 73.9 & 1.23 & $1690 \pm 5$ & 77.7 & 6.44 \\
\hline $7.71 \pm 0.01$ & $195 \pm 5$ & 70.0 & -4.11 & $1290 \pm 5$ & 78.6 & 7.67 \\
\hline $8.45 \pm 0.01$ & $165 \pm 5$ & 72.1 & -1.23 & $1065 \pm 5$ & 77.3 & 5.89 \\
\hline $9.71 \pm 0.01$ & $125 \pm 5$ & 70.5 & -3.42 & $700 \pm 5$ & 57.9 & -20.68 \\
\hline $10.16 \pm 0.01$ & $110 \pm 5$ & 76.7 & 5.07 & $680 \pm 5$ & 72.0 & -1.37 \\
\hline $10.39 \pm 0.01$ & $95 \pm 5$ & 72.8 & -0.27 & $610 \pm 5$ & 72.4 & -0.82 \\
\hline $10.85 \pm 0.01$ & $101 \pm 5$ & 75.1 & 2.88 & $695 \pm 5$ & 89.0 & 21.92 \\
\hline $11.01 \pm 0.01$ & $97 \pm 5$ & 72.8 & -0.27 & $605 \pm 5$ & 72.4 & -0.82 \\
\hline $11.45 \pm 0.01$ & $92 \pm 5$ & 76.2 & 4.38 & $565 \pm 5$ & 74.1 & 1.51 \\
\hline $11.66 \pm 0.01$ & $85 \pm 5$ & 70.5 & -3.42 & $580 \pm 5$ & 83.9 & 14.93 \\
\hline $11.78 \pm 0.01$ & $84 \pm 5$ & 71.7 & -1.78 & $550 \pm 5$ & 77.9 & 6.71 \\
\hline $11.79 \pm 0.01$ & $84 \pm 5$ & 71.7 & -1.78 & $550 \pm 5$ & 78.1 & 6.99 \\
\hline $12.03 \pm 0.01$ & $80 \pm 5$ & 71.0 & -2.74 & $455 \pm 5$ & 57.9 & -20.68 \\
\hline $12.10 \pm 0.01$ & $79 \pm 5$ & 70.9 & -2.88 & $520 \pm 5$ & 77.8 & 6.57 \\
\hline $12.79 \pm 0.01$ & $71 \pm 5$ & 71.0 & -2.74 & $460 \pm 5$ & 76.0 & 4.11 \\
\hline Average values & - & $72.7 \pm 2.0$ & $-0.46 \pm 2.74$ & - & $74.1 \pm 8.4$ & $1.56 \pm 11.53$ \\
\hline
\end{tabular}

Values of Young's modulus, $E_{1}$ and $E_{2}$, for the two resonances, as calculated, respectively, from Eqs. (11) and (12) are given along with relative percentage errors compared to the accepted value.

Fibre radius: $r=7.95 \pm 0.01 \mu \mathrm{m}$.

Fibre density: $\rho=2600 \pm 100 \mathrm{~kg} \mathrm{~m}^{-3}$.

Accepted Young's modulus: $E=73 \pm 2 \mathrm{GPa}$.

"theoretical" value of Young's modulus of $E$-glass fibres, viz. $73 \mathrm{GPa}$, for comparison.

The agreement is quite acceptable, although there is certainly experimental scatter, but this can hopefully be reduced with refinement of the technique.

Whether fundamental or secondary mode vibrations are exploited, the average values of Young's modulus established are in good agreement with the accepted value, with the average experimental values of $E$ being $72.7 \pm 2 \mathrm{GPa}$ for mode 1 and $74.1 \pm 8.4 \mathrm{GPa}$ for mode 2. However, it can be seen both from the standard deviations in Table 1 and from the scatter in Fig. 4, that the fundamental mode gives more precise results, possibly due to the relatively greater ease in identification of resonance. At its present stage of development, based on a visual technique for determination of the vibration mode, it is clear that 

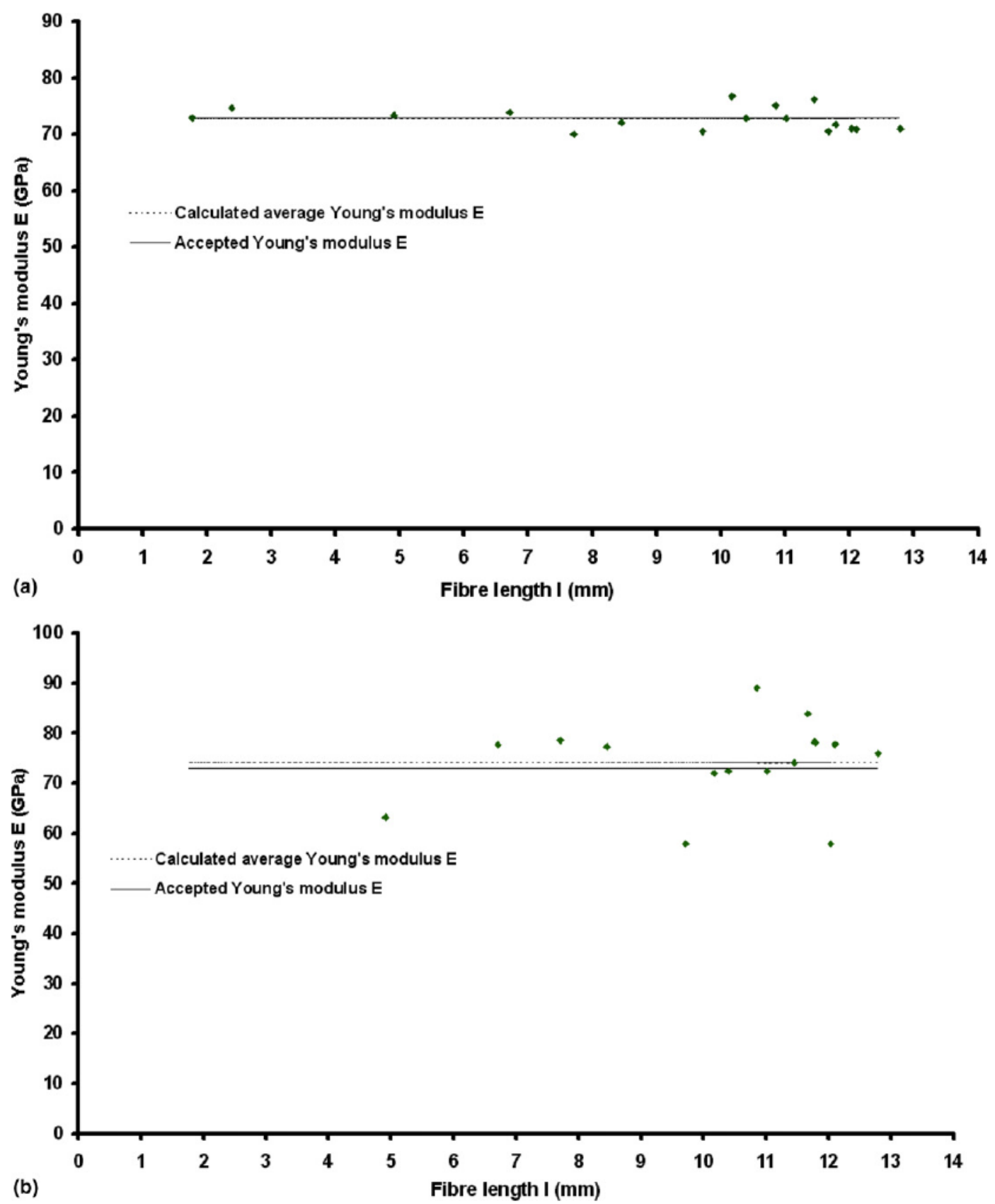

Fig. 4. Results of Young's modulus, E, vs fibre length from (a) fundamental, and (b) second vibration mode. The horizontal solid and dotted lines (virtually coincident in (a)) represent, respectively, the calculated average and accepted values of $E$.

characterisation of the second mode becomes more difficult at higher frequencies. Nevertheless, the present results show the promise of this novel technique. Furthermore, use of the technique would appear potentially useful for characterising other kinds of fibrous materials possessing a high elastic modulus (steel, aramid, carbon, etc.).

Having established the good correlation between the accepted value of $E$ and those obtained by the present method, when using virgin $E$-glass fibres, a study of aged sheet moulding compound fibres was undertaken. These fibres underwent various degrees of degradation or transformation during a recycling process, allowing them to be competitive with virgin materials. Data concerning the three recycling stages are summarised in Table 2. Only the fundamental vibration mode, $v_{1}$, was studied. The values for each stage of treatment correspond to the average and spread obtained from four samples.

Based on the results shown in Table 2, a strong decrease of Young's modulus is noted between the virgin and the reused fibres, this being particularly marked after the first step of shredding. The slight grinding involved provokes a loss of modulus of approximately $18 \%$, presumably because of the creation of local stresses within the fibres.

Since an acidic environment is present for the second step of the recycling process, a further, yet less significant, drop of approximately $7 \%$ is noted in comparison with the 
Table 2

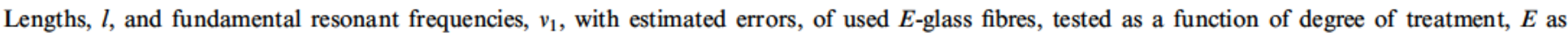
evaluated from Eq. (11), and relative percentage errors compared to the accepted value for virgin fibres

\begin{tabular}{|c|c|c|c|c|}
\hline Stage of treatment & Fibre length $l(\mathrm{~mm})$ & $v_{1}(\mathrm{~Hz})$ & $E$ of treated SMC fibres $\left(E_{\text {treated }}\right)(\mathrm{GPa})$ & $\frac{E_{\text {reated }}-E}{E} \times 100(\%)$ \\
\hline Shredding & $12.32 \pm 0.01$ & $71 \pm 1$ & $61.1 \pm 2.2$ & -18 \\
\hline Dissolving & $11.59 \pm 0.01$ & $76 \pm 2$ & $54.9 \pm 2.4$ & -25 \\
\hline Thermal treatment & $7.81 \pm 0.01$ & $168 \pm 2$ & $55.3 \pm 1.6$ & -24 \\
\hline
\end{tabular}

Fibre radius: $r=7.95 \pm 0.01 \mu \mathrm{m}$.

Fibre density: $\rho=2600 \pm 100 \mathrm{~kg} \mathrm{~m}^{-3}$

Accepted Young's modulus: $E=73 \pm 2 \mathrm{GPa}$.

first stage of shredding alone. The expected strong influence of the acid is probably partially counteracted by the chemical properties of $E$-glass fibres which resist an aggressive environment such as an acidic solution.

Thermal treatment of SMC fibres would seem to have little or no influence regarding the Young's modulus of the fibres. Within experimental error, the same value of Young's modulus is observed before and after the second and the third stages of the process. This is probably due to too low an isothermal temperature $\left(200^{\circ} \mathrm{C}\right)$ having negligible mechanical impact.

\section{Discussion}

The above description of the results shows the method developed here to be highly satisfactory for estimating fibre modulus. Some refinement may be necessary in order to reduce experimental scatter, but the potential of the technique is clear. However, at least part of the scatter may be intrinsic. This could be the case if the resonance peaks are too diffuse. A study of the shape of the resonance peaks is therefore of interest. Fig. 5 gives a schematic, yet typically semi-quantitative, representation of vibration amplitude vs frequency, arbitrarily centred on resonance, for simplicity. Three peaks are shown: that "expected", and those found both with a steel fibre support and with a polymeric fibre support, as used for the preliminary tests. The sharpness (or $Q$ factor) is less than anticipated in both experimental cases. Such a phenomenon of peak widening

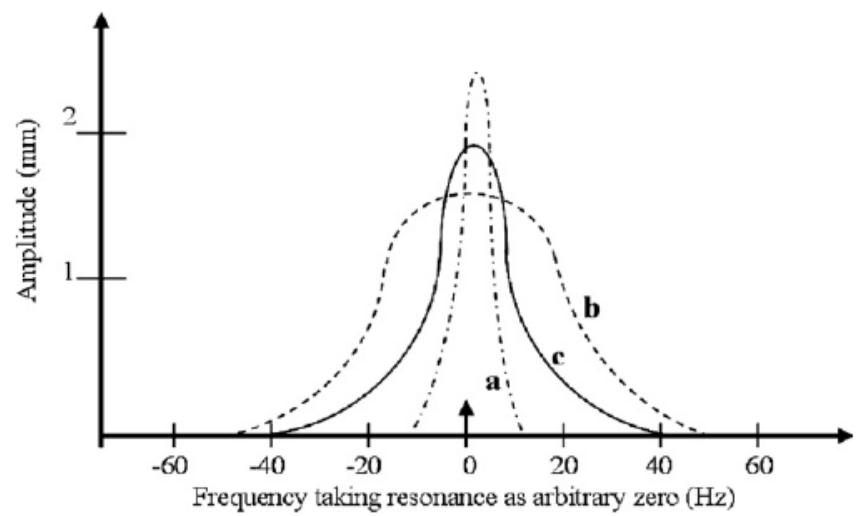

Fig. 5. Schematic representation of: (a) expected resonance peak shape, and those found with (b) fibre mounted on a polymeric support, (c) with fibre mounted on a steel support. is typical of some mechanism of energy dissipation occurring during oscillations which, without forced motion, would lead to (rapid) damping of the vibrations. In simple terms, the more significant the damping force, the wider is the peak. Fig. 5 therefore suggests that the cellulose acetate support is more dissipative than the steel support. As a consequence, identification of the resonant frequency becomes more difficult. This may be expected since the polymer will have an at least partially viscoelastic component to its mechanical behaviour, whereas the steel may be considered to be purely elastic under the conditions considered. In principle, the presence of a damping, or viscous, component shifts the resonant frequency to a lower value [19], but this is insignificant in the case studied, as will be seen from a simple calculation below. Notwithstanding, the resonance peak width, even for the steel support, is perhaps larger than may be expected intuitively. It is probable that the fibre itself may be treated as purely elastic, and is therefore not at the root of the problem. The adhesive "blob" is far more rigid than the fibre, especially in the direction of oscillation, perpendicular to the fibre axis, and thus may be discounted. Two possible causes are considered. Glass fibres are generally covered with sizing, a polymeric material applied to protect the glass surface (amongst other purposes). Considering a thin and homogeneous sizing layer of thickness, $\delta r$, on the fibre of radius, $r$, it can be shown that the effective fibre modulus becomes $\left(E+4 E_{\mathrm{s}}^{*} \delta r / r\right)$, where $E_{\mathrm{s}}^{*}$ is the (complex) Young's modulus of the sizing material. Taking $E_{\mathrm{s}}^{*}$ to be $1 \mathrm{GPa}$ (of which only a fraction will correspond to the loss modulus, $E_{\mathrm{s}}^{\prime \prime}$ ) and $\delta r$ as $0.1 \mu \mathrm{m}$, we find $4 E_{s}^{*} \delta r /(E r)$ at approximately $10^{-3}$. Thus, the viscous, or lossy, character endowed on the fibre, by the sizing, will be negligible.

Another possible explanation of the relatively wide resonance peak could be the air resistance acting on the oscillating fibre. The order of magnitude of the maximum fibre speed, $V_{\max }$, (at the fibre's free extremity, and when $y=0$ ) may be found from Eq. (2), taking $z(x)=z(l)(\approx 2 \mathrm{~mm}$, by observation), a fairly typical frequency of $100 \mathrm{~Hz}$, and setting the cosine term to unity, thus leading to a value of the order of $1 \mathrm{~m} \mathrm{~s}^{-1} . V_{\max }$ is thus well below the speed of sound in air and the latter may therefore be reasonably approximated to an incompressible fluid. The local (maximum value of) Reynolds number, $R e$, is given by $2 V_{\max } r \rho_{\text {air }} / \eta$, (where $\eta$ is the dynamic viscosity of air: $\approx 1.8 \times 10^{-5} \mathrm{~Pa} \mathrm{~s}$; and $\rho_{\text {air }}$ its density: $\approx 1.2 \mathrm{~kg} \mathrm{~m}^{-3}$ ), and is thus of order 1 . 
In this range of $R e$ (the following is apparently valid for $R e \geqq 100$, although perusal of a graph of $\log C_{\mathrm{D}}$ vs $\log R e$ suggests 10 may be a more reasonable cut-off value [19]), for a circular cylinder the drag coefficient, $C_{\mathrm{D}}$, is given approximately by $C_{\mathrm{D}}=24 R e^{-1}$ [20]. From the definition of $C_{\mathrm{D}}\left(=f_{\text {air }} /\left(\rho_{\text {air }} V_{\text {max }}^{2}\right)\right.$ for our example $)$, it may be readily shown that the typical maximum force per unit length of cylinder due to air resistance, $f_{\text {air }}$, during oscillation will be of order $12 \eta \cdot V_{\max }$. More generally, for any given local (in distance, $x$, and time, $t$ ) fibre speed, the damping prefactor is approximately $12 \eta$. In order to allow for air damping, we may modify Eq. (4) to give:

$y^{\prime \prime \prime \prime}+\frac{m}{E I} \ddot{y}+\frac{12 \eta}{E I} \dot{y}=0$.

A full analysis of Eq. (13) is, however, complex, and so instead, we present an order of magnitude approach. Simple static analysis of the beam (fibre) assuming constant loading per unit length leads to the expression:

$z(x)=z(l) \frac{x^{4}}{l^{4}}$.

The total force of air resistance over the fibre length, $F_{\text {air }}$, is then of order:

$F_{\text {air }} \approx 12 \eta \int_{0}^{l} \frac{x^{4}}{l^{4}} \dot{y}(l, t) \mathrm{d} x=\frac{12}{5} \eta l \dot{y}(l, t)$,

for any given value of $\dot{y}(l, t)$ in its sinusoidal cycle.

Assimilating, for simplicity, fibre oscillation with classic simple harmonic motion (SHM), it follows that the halfwidth on the frequency scale of the resonance peak at half maximum (squared) amplitude $[12,13]$ is given by $\left(F_{\text {air }} / \dot{y}\right) /(2 M)$, or approximately $\eta l / M$, where the mass, $M$, of a typical fibre of length of about $1 \mathrm{~cm}$ is $M \approx$ $\pi r^{2} l \rho \approx 5 \times 10^{-9} \mathrm{~kg}$. The half-width of the resonance peak is thus calculated at about $40 \mathrm{~Hz}$. Although seemingly rather high, this order of magnitude corresponds reasonably to that observed experimentally. Air resistance is thus a very plausible cause of the relatively wide resonance peaks observed, although not proved unequivocally. (An additional complication, in a more exact appraisal of this aspect, could be that local "disturbance" of the air in the zone repeatedly swept out by the fibre in motion may not be negligible, and could modify the damping pre-factor.)

A corollary of this simple SHM treatment is that the peak frequency for resonance should be slightly reduced due to damping. In the present context, where the natural frequency is $v$, the reduced frequency, $v_{\text {red }}$ is given by,

$v_{\mathrm{red}}=\left[v^{2}-\frac{18 \eta^{2} l^{2}}{25 \pi^{2} M^{2}}\right]^{1 / 2}$.

With the above values, we find that $v_{\text {red }} \approx 0.995 v$, so the effect of (air) damping on observed resonant frequency is small and any correction quite negligible. This corroborates the good agreement found between accepted and deduced values of $E$, as mentioned above.
Further work with fibres mounted in a reduced pressure environment could clarify certain aspects of these damping phenomena, although this procedure would reduce the simplicity of the method suggested here. Despite a lack of full understanding of the resonance peak width at present, it can nevertheless be seen that if the central values of frequency of the peaks are adopted, this novel experimental technique can be used to obtain very good estimates of Young's modulus for small diameter fibres, a parameter which is exceedingly difficult to obtain by other means. The technique could also prove to be very useful for studying fibres of limited length, such as natural fibres (e.g. pulp).

The above theory will apply to isotropic "beams" of circular section, such as glass, as studied, or metallic fibres. One drawback of the technique is the necessity to have fibres of regular cross-section, both from a geometrical and from a mechanical properties point of view. Otherwise, the basic theory above cannot be reliably applied (or, at least, its precision is reduced). A variable cross-sectional area and/or modulus would perturb the assumed constancy of the product, $E I$, in Eq. (4) et seq. in the following development. For example, some polymeric, and graphite fibres would suffer from this disadvantage. Notwithstanding, this same shortcoming can be levelled at direct tensile evaluation methods, where any changes in sectional characteristics will also perturb evaluation of fibre properties (evaluated moduli are effectively averaged). Any differences between compressive and tensile modulus should normally be negligible for the small strains involved.

Note, however, that a different, but constant, cross-section, coupled with a sufficiently isotropic fibrous material (in tension and compression), would only entail re-determination of the position of the neutral surface and recalculation of $I$, which is not a significant problem.

\section{Conclusion}

We have developed a novel and simple method for the evaluation of Young's modulus, $E$, of fine fibres, as often used to reinforce composite materials. Rather than submit fibres to tensile loads along their axis, they are retained only at one end and effectively "waggled" like a dog's tail! The fibre is mounted on an electrodynamic shaker, via a system of support, and by scanning frequency, zones of resonance can be found. Using simple beam theory, it is thus possible to calculate $E$. Using virgin $E$-glass fibres, of known characteristics, as a model, accepted and experimental values of Young's modulus were compared and the agreement was found to be very good. A significant reduction of Young's modulus was found with preliminary trials on initially similar fibres subsequently having undergone recycling treatments, such as shredding, dissolution or of a thermal nature. A phenomenon not yet totally understood, is the relatively large width of vibration resonance peaks, usually associated with energy loss. It is suggested that this is mainly attributable to dissipation caused by 
air resistance during fibre motion. The technique is hoped to be of use in the general assessment of fibres, and also for following their ageing behaviour.

\section{Acknowledgements}

Various people at our Institute have shown interest in, or have contributed, in their way, to this work, and we express our thanks, notably to B. Ayme, B. Gautier, P. Slangen and F. Spinelli. MERS, in particular, thanks J. Valy of the Centre des Matériaux of the Ecole des Mines de Paris for useful discussions.

\section{References}

[1] Cox HL. The elasticity and strength of paper and other fibrous materials. Br J Appl Phys 1952;3:72-9.

[2] Kelly A, Tyson WR. Tensile properties of fibre-reinforced metals: copper/tungsten and copper/molybdenum. J Mech Phys Solids 1965;13(6):329-50.

[3] Kelly A. Strong solids. London: Oxford University Press; 1973.

[4] Bunsell AR, Schwartz P. Fibre test methods. In: Carlsson L, Crane RL, Uchio $\mathrm{K}$, editors. Comprehensive composites materials. Oxford: Elsevier; 2000. p. 49-70.

[5] Deléglise F, Berger MH, Jeulin D, Bunsell AR. Microstructural stability and room temperature mechanical properties of the Nextel 720 fibre. J Eur Ceram Soc 2001;21(5):569-80.

[6] Andersons J, Joffe R, Hojo M, Ochiai S. Fibre fragment distribution in a single-fibre composite tension test. Composites Part B 2001; 32:323-32.
[7] Andersons J, Joffe R, Hojo M, Ochiai S. Glass fibre strength distribution determined by common experimental methods. Compos Sci Technol 2002;62:131-45.

[8] Montes-Moran MA, Young RJ. Raman spectroscopy study of highmodulus carbon fibres: effect of plasma-treatment on the interfacial properties of single-fibre-epoxy composites-Part II: characterisation of the fibre-matrix interface. Carbon 2002;40:857-75.

[9] Miyagawa H, Sato C, Mase T, Drown E, Drzal LT, Ikegami K. Transverse elastic modulus of carbon fibres measured by Raman spectroscopy. Mater Sci Eng A 2005;412:88-92.

[10] Miyagawa H, Kamiya D, Sato C, Ikegami K. Strain measurement for Raman-inactive substrates with $\mathrm{PbO}$ thin films using Raman coating method. J Mater Sci 1999;34:105-10.

[11] Kang CS, Maeda K, Wang KJ, Wakashima K. Dynamic Young's modulus and internal friction in particulate $\mathrm{SiC} / \mathrm{Al}$ composites. Acta Mater 1998;46(4):1209-20.

[12] Lee J, Kim SE. Free vibration of thin-walled composite beams with I-shaped cross-sections. Compos Struct 2002;55:205-15.

[13] Mathieu JP. Vibrations et phénomènes de propagation. T.1 Oscillateurs. Paris: Masson; 1974.

[14] Harris CM. Shock vibration. Handbook. London, Paris: McGrawHill; 1988.

[15] Ewins DJ. Modal testing: theory and practice, vol. 1. Taunton: Research Studies Press; 1995, p. 313.

[16] Benedetto AT. Tailoring of interfaces in glass fibre reinforced polymer composites: a review. Mater Sci Eng A 2001;302:74-82.

[17] Kia HG. SMC sheet moulding compounds science and technology. Hanser; 1993, p. 23 .

[18] Perrin D, Leroy E, Clerc L, Bergeret A, Lopez-Cuesta JM. Treatment of SMC composite waste for recycling as reinforcing fillers in thermoplastics. Macromol Symp 2005;221:227-36.

[19] Tritton DJ. Physical fluid dynamics. 2nd ed. Oxford University Press; 1988.

[20] http://www.aerospaceweb.org/question/aerodynamics/q0231.html. 